BULLETIN OF THE

AMERICAN MATHEMATICAL SOCIETY

Volume 77, Number 4, July 1971

\title{
THE PLEMELJ DISTRIBUTIONAL FORMULAS ${ }^{1}$
}

\author{
BY DRAGIŠA MITROVIĆ
}

Communicated by Peter Lax, December 9, 1970

1. Introduction. Let $\left(\varepsilon^{\prime}\right)$ be the space of Schwartz distributions with compact supports defined on $R$. Let $\left(\Theta_{\alpha}^{\prime}\right)$ be the space of distributions defined on the space $\left(\mathcal{O}_{\alpha}\right)$ of all infinitely differentiable complex-valued functions $f$ on $R$ such that $f(t)=O\left(|t|^{\alpha}\right)$ and $f^{(p)}(t)$ $=O\left(|t|{ }^{\alpha}\right)$ for all $p(|t| \rightarrow \infty)$.

In this announcement we extend the famous Plemelj formulas to the distributions in $\left(\mathcal{E}^{\prime}\right)$ or $\left(\mathcal{\Theta}_{\alpha}^{\prime}\right)$. A distributional extension in another direction has been given in [1]. The overlap with the present approach is little. The Plemelj numerically-valued relations are discussed in detail in [2], [5].

Paralleling the classical version, we will consider distributions $T$ that are contained in $\left(\mathcal{E}^{\prime}\right)$ or $\left(\mathcal{O}_{\alpha}^{\prime}\right)$ and define the generalized Cauchy integral of $T$ by $\hat{T}(z)=(1 / 2 \pi i)\left\langle T_{t}, 1 /(t-z)\right\rangle, \operatorname{Im}(z) \neq 0$.

2. Statement of results. In what follows, $D^{+}$and $D^{-}$denote the open upper and the open lower half-planes, respectively.

THEOREM 1. If $T \in\left(\mathcal{E}^{\prime}\right)$ and

$$
\hat{T}^{ \pm}(z)=\frac{1}{2 \pi i}\left\langle T_{t}, \frac{1}{t-z}\right\rangle
$$

for $z \in D^{ \pm}$, then $\hat{T}^{ \pm}=\lim _{\epsilon \rightarrow+0} \hat{T}^{ \pm}(t \pm i \epsilon)$ exist in $\left(D^{\prime}\right)$ and

$$
\begin{aligned}
& \hat{T}^{+}-\hat{T}^{-}=T, \\
& \hat{T}^{+}+\hat{T}^{-}=-\frac{1}{\pi i}\left(T * \operatorname{vp} \frac{1}{t}\right) .
\end{aligned}
$$

ThEOREM 2. If $T \in\left(\mathcal{\Theta}_{\alpha}^{\prime}\right)$ for $-1 \leqq \alpha<0$ and

$$
\hat{T}^{ \pm}(z)=\frac{1}{2 \pi i}\left\langle T_{t}, \frac{1}{t-z}\right\rangle
$$

AMS 1970 subject classifications. Primary 30A88, 46F10.

Key words and phrases. Distributions in $\left(\mathcal{E}^{\prime}\right),\left(\Theta_{\alpha}^{\prime}\right)$, convergence of distributions, decomposition of a distribution, generalized Cauchy integral, Plemelj distributional formulas, distributional analytic continuation, Hilbert boundary problem.

1 The results (with applications) presented in this note were delivered at the Oberwolfach Conference on Functional Analysis (October, 1970). 
for $z \in D^{ \pm}$, then $\hat{T}^{ \pm}=\lim _{\epsilon \rightarrow+0} \hat{T}^{ \pm}(t \pm i \epsilon)$ exist in $\left(\mathcal{O}_{\alpha}^{\prime}\right)$ and the formulas (1) and (2) are valid.

If $T \in\left(\mathcal{O}_{\alpha}^{\prime}\right)$ for $\alpha \geqq-1$ the same formulas are valid in (D').

THEOREM 3. If $T \in\left(\mathcal{O}_{\alpha}^{\prime}\right)$ for arbitrary fixed $\alpha$ and

$$
\hat{T}^{ \pm}(z)=\frac{k !}{2 \pi i}\left\langle T_{t}, \frac{1}{(t-z)^{k+1}}\right\rangle
$$

for $z \in D^{ \pm}$, then

$$
\begin{aligned}
& \hat{T}^{+}-\hat{T}^{-}=T^{(k)}, \\
& \hat{T}^{+}+\hat{T}^{-}=-\frac{1}{\pi i}\left(T^{(k)} * \operatorname{vp} \frac{1}{t}\right)
\end{aligned}
$$

in $\left(D^{\prime}\right)$. One supposes $1 /(t-z)^{k+1}=O\left(|t|^{\alpha}\right), \operatorname{Im}(z) \neq 0$.

THEOREM 4. In the above theorems the decomposition of distributions into the difference of two distributions is unique.

Proofs are based on the results in [3], [4] involving a criterion for a linear form to be a distribution, theorems on convergence of distributions and certain facts on the distributional analytic continuation. A complete account will appear elsewhere.

The announced theorems are applicable directly on some classical problems formulated in the sense of distributions in $\left(\mathcal{E}^{\prime}\right)$ and $\left(\mathcal{O}_{\alpha}^{\prime}\right)$ (for example, a Hilbert boundary problem for the half-planes, singuular convolution equations).

\section{REFERENCES}

1. E. J. Beltrami and M. R. Wohlers, Distributions and the boundary values of analyticfunctions, Academic Press, New York, 1966. MR 34 \#8172.

2. F. D. Gahov, Boundary-value problems, Fizmatgiz, Moscow, 1958; 2nd rev. ed., 1963; English transl., Pergamon Press, Oxford; Addison-Wesley, Reading, Mass., 1966. MR 21 \#2879; MR 27 \#6094; MR 33 \#6311.

3. D. Mitrović, Plemelj formulas and analytic representation of distributions, Glasnik Mat. Ser. III 3(23) (1968), 231-239. MR 38 \#4994.

4. - - Analytic representation of distributions in $\left(\Theta_{\alpha}^{\prime}\right)$, Glasnik Mat. Ser. III 5(25) (1970), 43-49.

5. N. I. Mushelišvili, Singular integral equations. Boundary problems of function theory and their application to mathematical physics, 3rd ed., "Nauka," Moscow, 1967; English transl. of 1st ed., Noordhoff, Groningen, 1953. MR 15, 434.

Institute of Mathematics, University of Zagreb, ZaGreb, Yugoslavia 